\title{
Peaking into the plant cell wall using cryo-FIB milling and electron cryo-tomography
}

\author{
William Nicolas ${ }^{1}$, Florian Fäßler ${ }^{2}$, Elliot Meyerowitz ${ }^{3}$ and Grant Jensen ${ }^{3}$ \\ ${ }^{1}$ Caltech - Meyerowitz and Jensen labs, United States, ${ }^{2}$ Institute of Science and Technology (IST) Austria - \\ Schur lab, Klosterneuburg, Austria, ${ }^{3}$ Caltech, United States
}

The plant cell wall is a complex cross-polylamellate pectocellulosic structure strong enough to withstand the high turgor pressure inside plant cells. Yet it can anisotropically yield to allow for directed cell expansion, thus dictating the shape of the cell. These properties are mostly due to the fibrillar nature of the cellulose fibers that organize in successive layers. They can dynamically reorient according to mechanical constraints making them a remarkable adaptive and resistant material. To better understand how the cellulose fiber network interacts with the other components of the cell wall, namely hemicelluloses and pectins and hereafter influences cell shape, white onion (Allium cepa) epidermal cell wall peels have been used this last decade to study the structure and mechanical properties of the primary cell wall, mainly by atomic force microscopy (Zhang et al. 2017 and Kafle 2013) and high magnification field emission scanning electron microscopy (Zheng et al. 2018). This technic allows to probe the orientation and state of the superficial layers of the cell wall. Despite groundbreaking insights on the functioning of the cell wall, our knowledge is mainly restricted to the few superficial layers, leaving the remaining layers, estimated in the hundreds, to be unaccounted for. To gain access to the whole depth of the cell wall and explore its structure in 3-dimensions, we applied cryo-Focused Ion Beam milling (cryo-FIB milling) followed by electron cryotomography (ECT). Abaxial cell wall peels from the outer-scales to innr-scales of white onion were generated. They are cut in small rectangles to fit on a Quantifoil grid and are laid with the internal face of the cell wall facing upwards. They are then plunge frozen in liquid ethane/propane and processed by cryo-FIB milling to prepare thin lamellae amenable for ECT study in the periclinal cell walls (C-E). For this first time, we were able to capture snapshots of the cell wall fibers and the surrounding compounds in a near-native, frozen hydrated cell wall in scales \#2, 5, 6, 7 and 8 in non-treated, pectate lyase and BAPTA (calcium chelator) treated conditions. So far, the two main findings of this work are 1) the very conserved bimodal distribution of the angle of the fibers $\left(-45^{\circ}\right.$ and $\left.+45^{\circ}\right)$ throughout the entire depth of the cell wall in all scales across the onion in the non-treated condition and 2) the ubiquitous presence of a mesh-like matrix bathing the fibers which we putatively associate to pectins because of its response to the pectate lyase treatment. 


\section{Sample preparation}

Abaxial cell wall peels from the 5th to 8th scale of white onion were generated according to ${ }^{2}(\mathbf{A})$. They are cut in small rectangles to fit on a Quantifoil grid and are laid with the internal face of the cell wall facing upwards. They are then plunge frozen in liquid vethane/propane $(\mathbf{B})$ and processed by cryo-FIB milling to prepare thin lamellae amenable for ECT study in the periclinal cell walls (C-E).

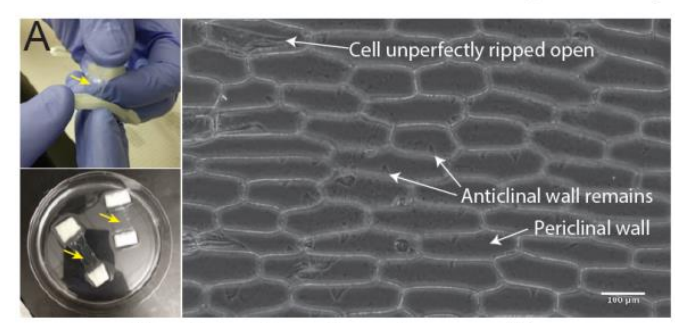

Abaxial cell wall peel generation

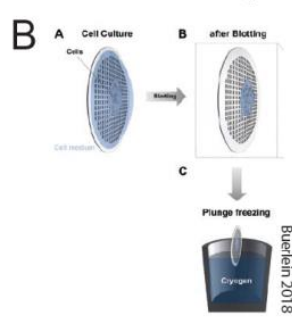

Plunge freezing

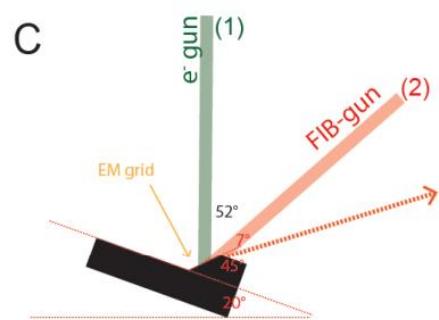

cryo-FIB milling process

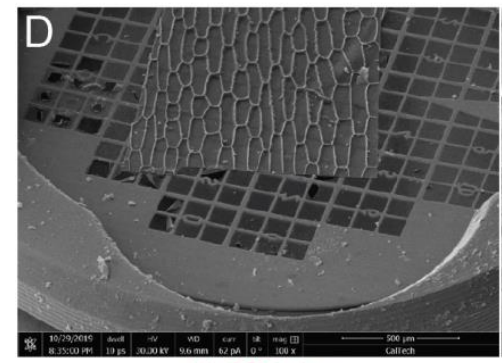

(1) SEM view

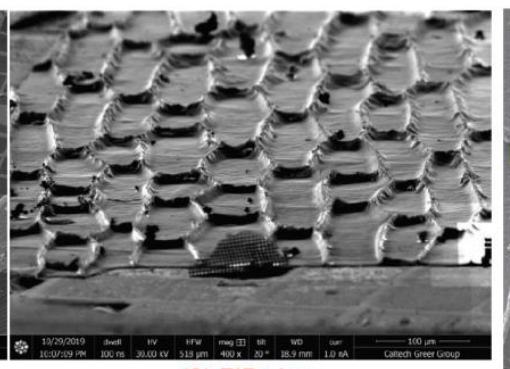

(2) FIB view
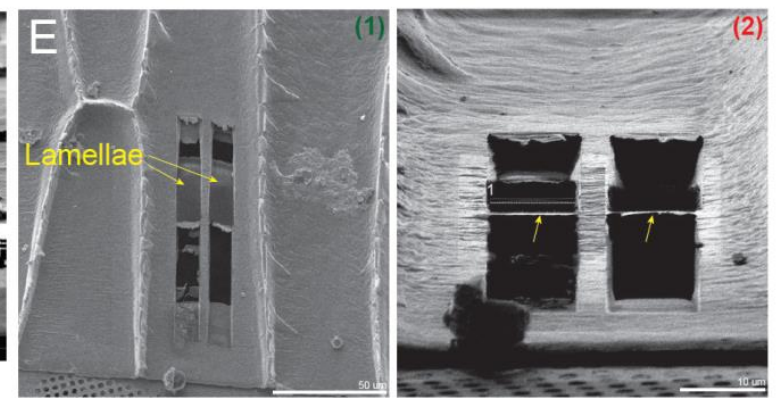

Figure 1. Figure 1: Figure abstract of the methodology used.

\section{Electron Cryo-tomography (ECT)}

Milling lamellae at an angle (usually around $10^{\circ}$ ) allows to sample the whole depth of the cell wall (A). Therefore, acquiring tilt series at increasing distances from the leading edge of lamella (coated in platinum) grants access to the different cell wall layers throughout the whole depth (B). 2D projections of the lamella allow the visualization of complex fibrillar networks (C) which can be better resolved in 3-dimensions, at high resolution, after tomographic reconstruction (Weighed$$
\text { A }
$$

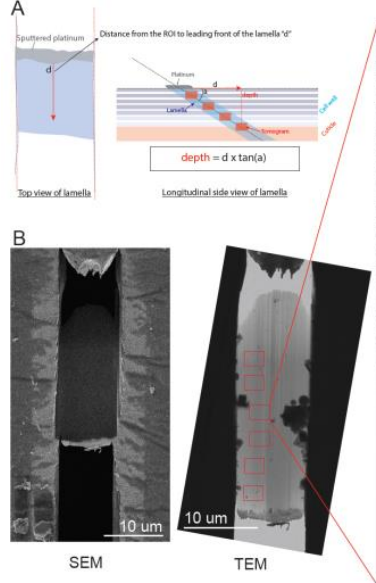
C. Tilt series acquisition

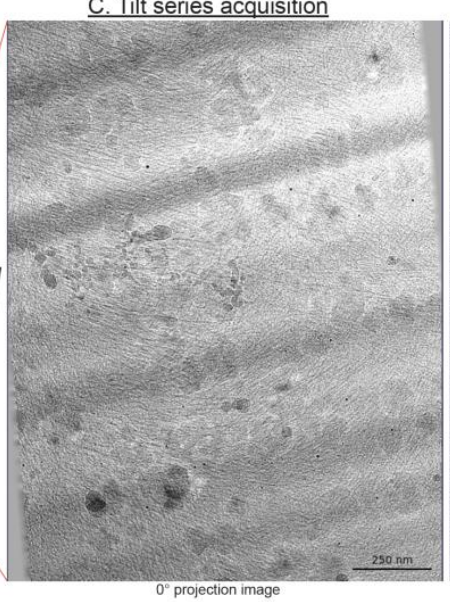
D. Tomographic reconstruction
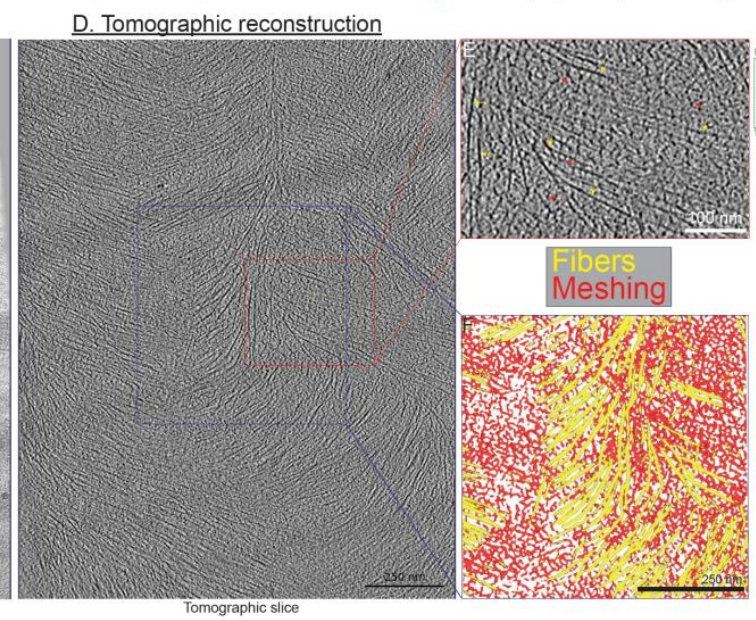
G. Fiber average

Figure 2. Figure 2: From mapping of the lamella to tilt series acquisition, tomogram reconstruction and segmentation.

\section{References}

Zhang T, Vavylonis D, Durachko DM, Cosgrove DJ. Nanoscale movements of cellulose microfibrils in primary cell walls. Nat Plants. 2017 Apr28;3:17056. doi: 10.1038/nplants.2017.56. Erratum in: Nat Plants. 2020 Dec;6(12):1504. PMID: 28452988; PMCID: PMC5478883. 
Kafle, K., Xi, X., Lee, C.M. et al. Cellulose microfibril orientation in onion (Allium cepa L.) epidermis studied by atomic force microscopy (AFM) and vibrational sum frequency generation (SFG) spectroscopy. Cellulose 21, 1075-1086 (2014).

Zheng, Y., Wang, X., Chen, Y., Wagner, E. and Cosgrove, D.J. (2018), Xyloglucan in the primary cell wall: assessment by FESEM, selective enzyme digestions and nanogold affinity tags. Plant J, 93: 211226. 